\title{
Article \\ Modeling of risk aversion linked to renewable energy policy and decision-maker behavior
}

\author{
Dieudonné Ecike Ewanga (D) \\ 1 University of Liège; dieudonne.ecikeewanga@doct.uliege.be
}

\begin{abstract}
This paper presents the behavior of decision makers, the possible choices and the strategies resulting from the uncertainties related to the integration of renewable energies. Its uncertainties are the risks associated with the volatility of renewable sources, the dynamics of energy production as well as the planning and operation of the electricity grid. The goal is to model the risk-averse decision-maker's behavior and the choice of integrating renewable energies into the electrical system. Following a bibliographic approach, we expose a methodology to model the decision-maker's behavior(risk aversion and predilection for risk) to risk taking. The risk-averse decision maker may adopt nonlinear utility functions. Risk aversion is a behavior that reflects the desire to avoid risk decisions and thus reduces the risk of adverse consequences. A decision support tool is provided to the decision-maker to choose a best-fit strategy based on his preferences. The rational and risk-averse decision-maker would seek to maximize a concave utility function instead of seeking to minimize its cost. Taste or aversion to risk can be modeled by a thematic function of utility.
\end{abstract}

Keywords: Modeling,risk averse, decision maker,choice,strategy,utility

\section{Introduction}

Particular attention in recent years has been paid to the security of supply, reliability and robustness of electricity systems while integrating renewable energy resources. Given the random nature of renewable energies, the decision to integrate them into the electricity system or not is similar to a risk exposure. Decision maker is interested in knowing if taking a decision under uncertainty is likely to result in a consequence. Then, if it is important to choose another appropriate decision. This is a risky future decision whose probabilities are known. Decision analysis based on probabilities are discussed in [1]. Theory of utility and decision are developed to characterize behavior in situations of risk. Decisions in engineering are invariably caught in a subtle uncertainty [2] [1] about the costs and the current and future intervention of the system, including the reactions associated with low probability and high consequence events. However, choice theory in uncertainty aims to provide a coherent framework of principles of rational behavior to analyze and guide decision-makers attitudes to potential loss/benefits.

This is traditionally studied on a lottery space or random variables while the evaluation of results and corresponding probabilities ultimately depends on the preferences of the decision maker [3]. Risk-based methods for reliability investments in electrical power distribution systems are discussed [4]. Risk-based methods are proposed [4]. They take into account extreme outage events in risk assessments incorporating impact and estimating the total probability distribution of total reliability. The framework deals with the decision in risk from a normative perspective, that is to say, where one wonders about the models of preferences likely to lead to a "rational behavior". This decision is based on the assumption that the expected value of utility is the appropriate decision criterion. A common approach when making decisions is to base them on the expected values. The expected value (average) is an operation that multiplies the consequence of each event by its probability and sum over all possible events. By using the expected value, a high frequency event with low consequences will have the same weight as a low frequency event with consequences high 
(if the products are the same). Basing the decisions on the average values of the results of the simulation corresponds to the adoption of a strategy without risk. The choice of the decision-maker depends on his behavior and his preferences [5] [6].

The decision maker can use different risk strategies to see how the results vary. The three behaviors that decision makers face are predilection, neutrality, and risk aversion. So it is a question of using prediction modeling and/or risk aversion in a context of choice. We seek to help decision makers make the choice by taking into account their risk behavior.

The decision maker chooses the solution that maximizes its contentment, in other words the expectation of its utility function. The expected utility model is introduced by von Neumann and Morgenstern [7] [8] [9] [10]. The theory of the decision gives the means to the decision-maker not only to analyze his problem but also to be able to justify whether the proposed solutions are rational. A rational and risk averse decision-maker seeks to maximize a utility function [11] concave rather to seek to minimize the cost.

The idea of this model is to model the choices available to the decision maker as a set of consequences associated with a distribution of probabilities. The decision maker makes a choice between these consequences based on their respective probabilities and risk behavior. Decisions can be multicriteria [9]. In [12] modeling uncertainty of induced technologycal change. Three features are introduced: (i) increasing returns to scale for new technologies; (ii) clusters of technologies that induce learning of the energy system; and (iii) a decision-making approach to help policymakers prioritize [13]. Rapid deployment of renewable energy sources for diversified energy production is a topical issue discussed [14].

A new multi-criteria decision support system to help calculate the most appropriate set of energy options to provide sufficient energy and meet local demands for improved livelihoods is discussed [15].[16] indicates that existing energy decision support tools have been useful but are mostly incomplete. The decision criteria for investing in the integration of these sources are economic, technical and environmental decisions. Economic decision analysis uses criteria such as Net Present Value (NPV), Return on Investment (ROI) and Pay-Back Time (PBT). The analysis of engineering decisions is based on cost and efficiency. In [17] the ELECTRE III method is used as a multi-criteria analytic support to identify optimal investment decisions from a predefined set of solutions. In [18] the process analysis technique (AHP) is used to quantify the various differences of opinion and uncertainties in the hybrid system performance plans (Wind and PV).

A new system of multi-criteria decision support to help compute the most appropriate set of energy options to provide enough energy to meet local demands that improve livelihoods is discussed [19]. In this article one introduces the model of the policy of aversion to characterize the behavior of the decision maker. [20] discusses decision making for the operation, maintenance and [21] investment planning of power systems in the face of uncertainty.

This is a policy to clarify the advantages or disadvantages that are difficult to discover by the present value expected. This is a policy to clarify the before or disadvantages that are difficult to discover by looking only at the expected net present value. Risk aversion is at the heart of economic and financial thinking [22]. The notion of risk aversion considered in this context is different from the more well-known risk, as the product of a probability of occurrence of an event by its consequences.

The risk aversion is a behavior that reflects the desire to avoid any decision of risk, and thus which reduces the probabilities of adverse consequences. The model is translated by the utility curve which is the expression of the decision-maker's behavior. Utility values are evaluated in such a way that the decision maker's risk behaviors are automatically incorporated into a standardized scale between 0 and 1 . Utility is a real-world value of the possible outcomes of decisions, taking into account the preferences of the decisionmaker. This is a subjective measure of the value of the specific results of the decisions taken, or the degree of satisfaction of the decision-maker with the benefits realized [23].

The objective is to model the aversion to the choice of a strategy and/or policy leading to the deployment of renewable energy to reduce the probability of an accidental failure 
occurring during the operation of the system power supply. This paper is organized as follows. Section 2 describes the methodology framework. Section 3 introduced a application of integrating renewable energy. Section 4 presented a sensibility analysis with $\lambda$ parameter. Section 5 conclusion and discussion of this paper.

\section{METHODOLOGY}

The methodology is based on the principles and axioms of the existence of the expected utility and takes into account the preferences faced with an uncertain situation based on the assumption.

\subsection{Assumption}

In this context assumes that decision makers maximize the expected value of their investment.

\subsection{Formalization of risk taking}

The von Neumann and Morgenstern [3] utility theory of choice under uncertainty represents one of the major pillars of modern decision making science, and plays a fundamental role in economics, finance, operations research, and other related fields[? ]. Conditions of the preferences must be satisfied to be represented by an expected utility function. However, the definition of a lottery preference relationship is sufficient in itself to characterize the decision-maker's risk behavior. Why the expected utility, rather than the expected value, is better the criterion of choice. For there to exist a function $u(x)$ of utility $u$ and of consequence $x$, the relation of preference should check axioms von Neumann and Morgenstern (1944). Let $r, p, q$ three lottery distributions in which the decision maker must make the choice, related to the preference he gives to each of the distributions. The decision maker must check some axioms:

\section{- comparability:}

$\diamond \quad p>p$ : " $p$ is preferred strictly to $q$ ", the related utility function is such that $u(p)>u(q)$

$\diamond \quad p \sim q: " p$ is indifferent to $q "$, so $(p>p$ and $q>p)$ and $p \succsim q: " p$ is preferred or indifferent to $q "$

- Independence and/or substitution:

$$
\diamond \quad \forall p, q, r \in \mathbf{L}, \alpha \in[0,1], p>q \Rightarrow \alpha p+(1-\alpha) r>\alpha q+(1-\alpha) r
$$

\section{- Continuity:}

$$
\diamond \quad \forall p, q, r \in \mathbf{L} \alpha \in[0,1], p>q>r \Rightarrow \alpha p+(1-\alpha) r>q \text { and } q>\alpha q+(1-\alpha) r
$$

where $\mathbf{L}$ is the set of lottery. If the axioms are checked and accepted by the decisionmaker accepts, there is a utility function $u: \mapsto \mathbb{R}$, such that $\forall A_{1}, A_{2} \in \mathbf{L}(\mathbf{x})$ where $x$ is the set of consequences of a decision problem, where $\mathbf{L}(\mathbf{x})$ finished lotteries. We note $A=[p x(1-p) y]$ the lottery to win $x$ with a probability $p$ and $y$ with probability $(1-p)$. After comparing two lotteries, the decision maker prefers $A_{1}>A_{2}$. We deduce $A_{1}=$ $\left[p_{1} x_{1} ;\left(1-p_{1}\right) y_{1}\right]$ and $A_{2}=\left[p_{2} x_{2} ;\left(1-p_{2}\right) y_{2}\right]$. Formally, there is a $u$ utility function on the consequence set such that,

$$
p_{1} u\left(x_{1}\right)+\left(1-p_{1}\right) u\left(y_{1}\right)>p_{2} u\left(x_{2}\right)+\left(1-p_{2}\right) u\left(y_{2}\right)
$$

that is, the utility value difference of $A_{1}$ is greater than the expected utility of $A_{2}$. The utility expectations of the winnings of the various lotteries are thus compared with the utility of the expectation of the gains that these lotteries allow, by representing the different behaviors by the utility curves. For probability calculations we rely on Weibull law. It models in many situations and characterizes the behavior of the system. 


\subsubsection{Utility theory and stochastic dominance}

The von Neumann-Morgenstern utility theory argues that when the preference relation $\geq$ of the decision maker satisfies certain axioms above. Il existe une fonction $: \mathbb{R} \rightarrow \mathbb{R}$, telle qu'un résultat $X$ est préféré au résultat $Y\left(\right.$ " $\left.X \geq Y^{\prime \prime}\right)$ if and only if,

$$
\mathbb{E}[u(X)] \geq \mathbb{E}[u(Y)]
$$

Thus, in effect, a decision making problem under uncertainty for a rational decision maker reduces to maximization of his/her expected utility: $\max \mathbb{E}[u(X)] \mid X \in \mathcal{X}$.

\section{Modeling risk aversion in utility theory}

There are some well-known means of risk aversion in the literature such as meanvariance, value-at-risk, expected shortfall, conditional value-at-risk, and second-order stochastic dominance [? ][? ]. The purpose of the expected utility model is to search for the maximum value [14]. The decision-maker's behaviors are evaluated by his/her utility expectancy (and not his mathematical esteem) [24] Consider a lottery, that is, a probability distribution $p_{i}$ on a set of consequences $x_{i}$ of a die for example from a finite number of consequences $x_{i} \in \mathbb{R}$, knowing that die probabilities are known in advance. Suppose there are $X_{i}$ consequences and whose values are represented by $x_{1}, x_{2}, \cdots, x_{n}$ attached to the probabilities $p_{i}$ represented by $p_{1}, p_{2}, \cdots, p_{n}$ where $0 \geq p_{i} \geq p_{1}$ for $i \in 1,2, \cdots N$ and $\sum_{i=1}^{n} p_{i}=1$. The consequences here are monetary sums.

Risk aversion is therefore a behavior that is revealed in situations where potential wealth is high but at risk. The terms $x_{i}$ represent the consequences in terms of gain and/or loss and $p_{1}, p_{i}$ denote the probabilities of winning and losing respectively for the case of random sorting. The question is how can one model the risk taking? The example below illustrates the case of risk taking where the utility function characterizes the decisionmaker's behavior when choosing between two perspectives. Or an initial wealth of 50 .

Two perspectives (lotteries) are possible, $\mathbf{L}_{\mathbf{1}}$ and $\mathbf{L}_{\mathbf{2}}$. Lottery, $\mathbf{L}_{\mathbf{1}}$ has a consequence of 150 with a probability equal to $\mathbf{1}$. The lottery $\mathbf{L}_{2}$ gives a $p$ probability equal to 0.5 of gaining a consequence of 300 with a probability $(1-p)$ equal to 0.5 of losing with a consequence of 0 . The decision-maker's behavior is modeled by the utility function $u(x)=x^{2}$. $\mathbf{L}_{1}$ is a utility of $1 *(150)^{2}=22500$ and the lottery $\mathbf{L}_{2} 0.5 *(300)^{2}+0.5 *(0)^{2}=45000$. Figure 1 below illustrates the risk-taking decision from which the decision-maker's behavior is shown in figure 2 below.

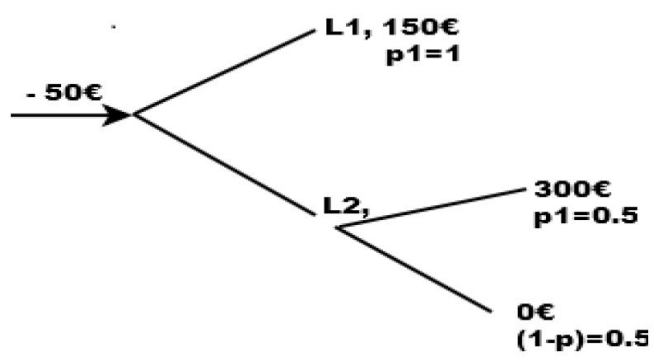

Figure 1. This figure represents a decision problem in which the decision maker must make the choice according to his preferences. The choice to be made between a risky alternative and a certain alternative in the lottery perspective of monetary gains

With regard to figures 1 and 2, are we ready to participate in such a lottery? However, the two alternatives are such that, on the one hand the player can keep his wealth his initial wealth and refuse to take part in the lottery that could win him easily but that could also make him lose everything. His gain a certain and therefore without risk. On the other hand, the player can agree to participate in the lottery to increase his final wealth. The term decision maker means player, network manager, service, $\cdots$. The proposed model uses the exponential utility function of the following form (2),

$$
u(x)=1-\exp (-\lambda \cdot x)
$$

\section{.}




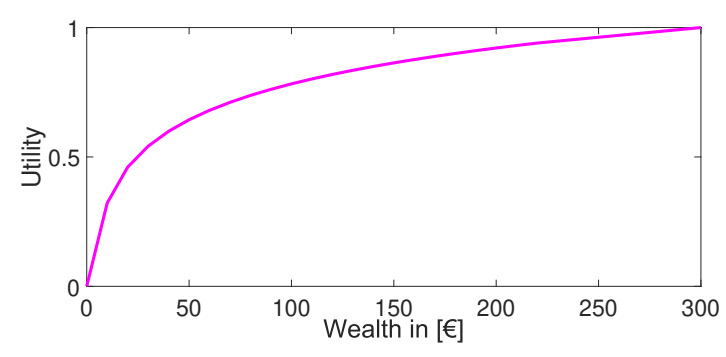

Figure 2. Utility curve

to which is associated the utility expectancy,

$$
\begin{aligned}
E(u(x))=p_{1} \cdot\left(1-\exp (-\lambda \cdot x)+p_{2} \cdot(1-\exp (-\lambda \cdot x)\right. \\
E(u(x))=\begin{aligned}
& p_{1} \cdot u\left(1-\exp (-\lambda \cdot x)+p_{2} .\right. \\
& (1-\exp (-\lambda \cdot x) \cdot u(1-\exp (-\lambda \cdot x)
\end{aligned}
\end{aligned}
$$

where $\lambda$ is a risk aversion parameter obtained by the ratio between second and first derivatives such that $\lambda=-\frac{u^{\prime \prime}(x)}{u^{\prime}(x)}=-\frac{\lambda^{2} \cdot \exp (-\lambda \cdot x)}{\lambda \cdot \exp (-\lambda \cdot x)}>0$. This constant and positive ratio is a coefficient of Arron and Pratt. As a result, the utility can be increasing, $\left(u^{\prime \prime}(x)>0\right.$ respectively concave with a positive and convex coefficient, $\left(u^{\prime \prime}(x)<0\right.$ with a negative coefficient. the correspondence between the form of the utility function and the behavior of the decision-maker equation (2) by the term $\frac{1}{\lambda}$ and we have $u(x)=\frac{1-\exp (-\lambda . x)}{\lambda}$.

We exploit a function is proposed [25] and implemented by [26] where the concave curve represents the gains and the convex curve for the losses and more steep for losses than gains. The general form of the exponential utility function used (5) for the strategies/lotteries proposed, allows to define the negative values of costs by determining all the behavior of the decision maker(5),

$$
u(x)=\frac{1}{1-\exp ^{-\lambda}} \cdot\left[1-\exp ^{-\lambda} \cdot\left(\frac{C_{\max -X}}{C_{\max }}\right)\right]
$$

where $C_{i}$ denotes the different costs in for all policies/lotteries $X_{i}$ such as the vector $X_{i}\left[C_{1}, \cdots, C_{i}\right]$. The expected utility associated with both strategies is defined such that,

$$
E\left(x_{1}\right)=\frac{1}{1-\exp ^{-\lambda}} \cdot\left[1-\exp ^{-\lambda} \cdot\left(\frac{C_{\max -E_{X 1}}}{C_{\max }}\right)\right]
$$

and

$$
E\left(x_{2}\right)=\frac{1}{1-\exp ^{-\lambda}} \cdot\left[1-\exp ^{-\lambda} \cdot\left(\frac{C_{\max -E_{X 2}}}{C_{\max }}\right)\right]
$$

where the $E_{X i}$ are the expected values of the strategies. Let $A=E(u(x))$,the utility expectations $\mathrm{E}(\mathrm{u}(\mathrm{x}))$ of two policies are determined by maximizing the utility function as follows,

$$
\begin{aligned}
A= & p_{1} \cdot \frac{1}{1-\exp ^{-\lambda}} \cdot\left[1-\exp ^{-\lambda} \cdot\left(\frac{C_{\max -C_{1}}}{C_{\max }}\right)\right] \\
& +, \cdots,+p_{i} \cdot \frac{1}{1-\exp ^{-\lambda}} \\
& \cdot\left[1-\exp ^{-\lambda} \cdot\left(\frac{C_{\max -C_{i}}}{C_{\max }}\right)\right]
\end{aligned}
$$


Table 1. System strategy

\begin{tabular}{l|c|c|c|c|}
\hline P.risk averse & & $\lambda=\mathbf{3}$ & & $\lambda=-\mathbf{3}$ \\
\hline Strategy & $A_{1}$ & $A_{2}$ & $A_{1}^{\prime}$ & $A_{2}^{\prime}$ \\
\hline $\mathbf{u}(\mathbf{E}(\mathbf{x}))$ & 0.9250 & 0.9674 & 0.4185 & 0.4626 \\
$\mathbf{E}(\mathbf{u}(\mathbf{( x ) )}$ & 0.8869 & 0.9489 & 0.5278 & 0.4835 \\
\hline
\end{tabular}

where $u(E(x))$ and $E(u((x))$ indicate expected utility and expectation of utility respectively. In the context of the theory of utility and decision-making in risk situations, the choice is a function of preferences. The decision-maker analyzes the various possible choices and chooses the one that maximizes its utility. In [27] the renewable energy investment problems and formulation are discussed. Let an investor (decision-maker) who wants to invest in renewable energy projects e.g PV panels and the wind turbine for $T$ horizon. At each stage of the project, the investor must make a decision whether to invest or not. The objective is to maximize the return generated by the investment.

\section{Application:integrating Renewable Energy}

Investment in energy production from renewable energy technologies can be considered as an assurance of supply of electricity. The expansion of renewable energy production [28] involves decisions to compare the alternatives and the degree of uncertainty [11] which is presented. Some elements on the taking of Renewable energy integration decisions are discussed [14]. The context of this application is presented in the choice integrating renewable energy into the grid as a lottery with a random gain in the form: Lottery is a probability distribution $p_{i}$ on a set of consequences $X_{i}$ (here the consequences are the costs.

$$
X_{i}= \begin{cases}p & : \text { proba that grid is working } \\ 1-p & : \text { proba that grid has at } 1 \text { fault }\end{cases}
$$

with the degree of uncertainty that results from barriers and economic barriers and techniques related to cost and technological immaturity respectively and especially to the production capacity. The terms $p$ and $1-p$ indicate the probabilities of good functioning and failure of the existing network. $p^{\prime}$ and $1-p^{\prime}$ denote probabilities of smooth operation and failure in the case of renewable energy integration. Denote by $X_{c, f}, X_{c, d}$ and $X_{p, f}, X_{p, d}$, network operation and fault and renewable energy integration respectively. The indices $d$ and $f$ indicate the fault and the operation. The term $R(t)=p$ represents the probability that the network has worked without failure at time $t$ and $1-R(t)=1-p$, the probability that the network will know at least one accidental fault. The observation time is 24 hours.

The optimal choice between the two strategies would be the one that maximizes the utility expectancy $E(u(x))$ taking into account the behavior of the decision-maker with respect to risk. However, the decision maker's choice depends on (i) the preferences on the consequences in (gain and/or loss). These consequences represent the costs (network fault and investment on the integration of renewable energy),(ii) risk and therefore laws of probability associated with different consequences, (iii) the behavior of the decision maker.

Two strategies (lotteries) are possibles. One represents the network (with failures and the other is the network+integration of decentralized sources (e.g PV and battery storage) to increase the production of energy. Figure 3 below represents the two lotteries where the decision-maker must choose between network(if he prefers to use corrective in case of failure), or network+renewable energy(to prevent accidental breakdowns and ensure good operation during peak hours). The values of expected utility and utility expectancy are shown in table 1 below.

Risk taking is a lottery, that is, a discrete distribution of probabilities $p_{i}$ on a set of consequences $x_{i}$. The utility of participating in such a St.Petersburg-based lottery does not equate with the usefulness of the mathematical expectation of lottery winnings, but with 


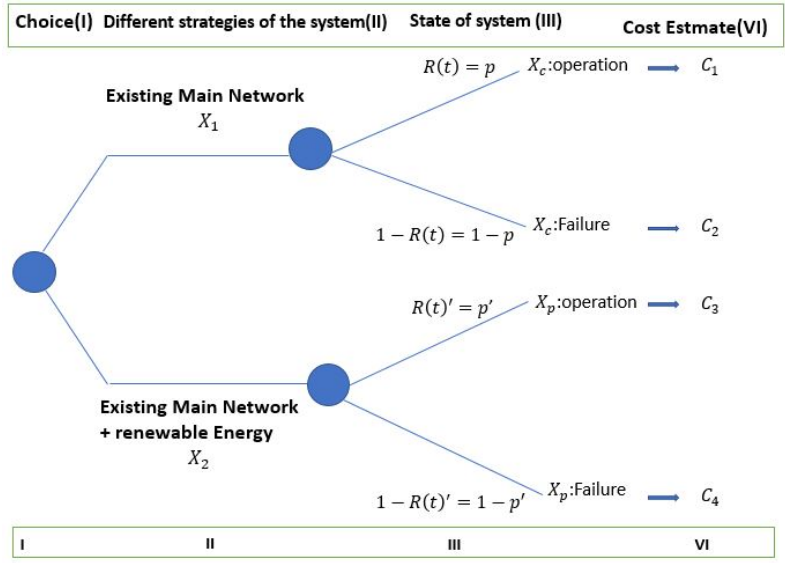

Figure 3. Lottery application model

that of the utilities contributed by the winnings. The expectation of rationalization some choices, but doesn't account for certain behaviors.

The goal is to allow the decision maker to choose between the two lotteries that which maximizes the expectancy of the utility function. For each of the strategies, are associated the different consequences $x_{i}$ in terms of the monetary costs in which the different probabilities are affected $p_{i}$. The decision maker makes the decision by comparing the two strategies $A_{1}$ and $A_{2}$ according to the one that maximizes its utility.

According to the calculation, the utility expectation $E(u(x))$ is less than the expected utility $u(E(x))$ for the strategy $\left(A_{1}\right)$ such that $E\left(u\left(X_{1}, X_{2}, X_{3}\right)>u\left(E\left(X_{1}, X_{2}, X_{3}\right)\right.\right.$ for the $A_{2}$ strategy, expected utility $u(E(X)$ is greater than the expected utility $E(u(x))$ such that $E\left(u\left(X_{1}, X_{2}, X_{3}\right)<u\left(E\left(X_{1}, X_{2}, X_{3}\right)\right.\right.$.

Let $X_{i}$ consequences inlinked to the lottery, $X_{i}[0,11400,22800]$.

Get the expected values $E(u(x))$ of two lotteries are [7836,and 4411.4] respectively with max cost $C_{\max },[25800]$ taken into account for a given system with the aversion parameter $\lambda=3$. Figure 4 below shows the decision maker's behavior. The mauve color indicates the risk-averse behavior for $\lambda=3$, while the green color presents the behavior of a decision-maker who has the taste of the risk (predilection) for $\lambda=3$.

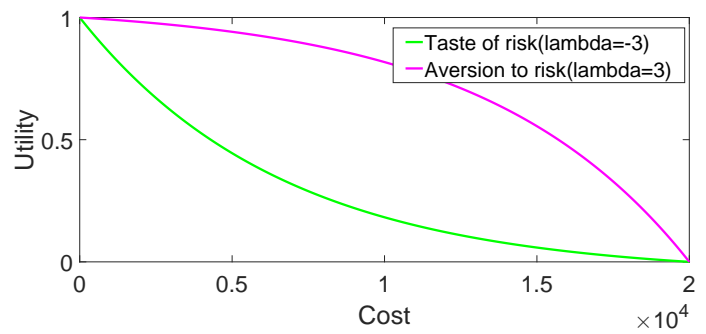

Figure 4. Attitudes towards risk

The decision-making model in risk is based on maximizing the expectation of utility function. The goal is to represent the decision-maker's behavior regarding risk taking. Two types of behavior are to be distinguished: risk aversion and risk predilection. The behaviors of the decision maker are translated by the curves of utility, concave, convex and nonlinear. In the following section the sensitivity analysis of the parameter lambda presented (6) to observe the choice of the decision maker and also the curves of utility.

\subsection{Discussion vs sensitivity analysis with $\lambda$ parameter}

Two groups of policies are considered with two risk-taking strategies for decision making: network $\left(A_{1}, A_{2}, A_{3}, A_{4}\right)$ and network+renewable energy $\left(A_{1}^{\prime}, A_{2}^{\prime}, A_{3}^{\prime}, A_{4}^{\prime}\right)$ following figures 5 and 6 . In each policy/lottery is associated with the consequences $X_{A 1}$ 
Table 2. Strategy $A_{1}, A_{2}$ and $A_{1}^{\prime}, A_{2}^{\prime}$

\begin{tabular}{l|c|c|c|c}
\hline P.risk averse & & $\lambda=\mathbf{1}$ & & $=-\mathbf{1}$ \\
\hline Strategy & $A_{1}$ & $A_{2}$ & $A_{1}^{\prime}$ & $A_{2}^{\prime}$ \\
\hline $\mathbf{u}(\mathbf{E}(\mathbf{x}))$ & 0.8271 & 0.8421 & 0.6378 & 0.6624 \\
$\mathrm{E}(\mathbf{u}(\mathbf{( x ) )}$ & 0.8119 & 0.8309 & 0.6637 & 0.6246 \\
\hline
\end{tabular}

and $X_{A 2}$ in such that $[0,1000,2000]$ and $[500,1500,2500]$ respectively following figures 5 and 6 . For each lottery there are consequences inand probabilities of good functioning of the network and the probabilities of having at least one breakdown such as: $[0.37,0.59,0.04]$, and $[0.9,0.09,0.006]$. After calculation, we obtain the expected utility values $u(E(u))$ and the expectation of utility $E(u(x))$ including $[0.8271]$ and $[0.8119]$ for lottery $A_{1}$ with max $\operatorname{cost} C_{\max }$ equal to [670] on the one hand and [0.8421] and [0.8309] on the other hand associated with the lottery $A_{2}$ with $C_{\max }$ equal to [600].

We want to observe the choice of the decision-maker and also his behavior in the face of risk taking across the curves of 'utilities. Indeed, this sensibility analysis is carried out thanks to the aversion parameter $\lambda$, fixed such that $\lambda=1$,resp.-1 (table 2 ) and $\lambda=2$, resp.-2 (table 3).

A utility curve may include elements(distinct behaviors) of all the curves shown in figure 5. For $\lambda=2$, resp. $\lambda=1$, the concave curves attests a decision maker with an aversion to risk. The decision maker prefers to work the network by doing the fix (i.e the network works until it goes down and then take the action to make the fix).

For $\lambda=-2$, resp. $\lambda=-1$, the convex curves attest to a decision-maker with a predilection for risk. The decision maker agrees that the network works with the support of renewable energy to prevent breakdowns and peak loads. The concave utility curve has diminishing marginal utility, is associated with risk-averse behavior (risquophobia), while the convex curve exhibits an increasing marginal utility reflecting a risky (risky) risk behavior. The logic behind this is that, in the case of risk aversion, the decision-maker associates a lower utility with a risky event than an event that provides with certainty the same expected value. Conversely, for a risk-loving decision-maker, the expected utility of a risky event will be greater than the utility of obtaining some expected value.

Definition of an appropriate energy policy is essential to guarantee the production of quality electrical energy at minimum cost. In most cases, decision-making is complex because, in addition to the difficulty of making a choice among others, the decision-maker has to deal with uncertainty. Decision-maker's decision support tool is presented. The decision-maker's behavior and acceptability for the investment are discussed to find a trade-off between renewable generation, investment and operational risks and the economic impact. Investing in renewable production is a choice. The decision-maker, whether he or she manages the electricity grid, makes his choice based on preferences, the appearance of breakdowns/load shedding and cost reduction. This leads to establishing an expected utility model by querying the decision maker about preferences, and modeling the behavior using mathematical utility functions. The choice of the strategy is therefore done by maximizing the expectation of the utility of the decision maker.

The presented model can be applied to any strategy, if one can quantify the consequences and their probabilities of occurrence. Choosing under uncertainty provides the coherent framework of rational behavior principles to analyze and guide decision makers attitudes to potential loss/benefits. The concave utility curve has diminishing marginal utility, is associated with risk-averse (risky) behavior, while the convex curve exhibits an increasing marginal utility translating risk (risky) risk behavior.

\section{CONCLUSIONS}

The objective of the work is, knowing the decision maker, the systems (for example the different technologies of energy production) and associated costs as well as the corresponding probabilities, to calculate the utility expectancies. The use of expected utility model allowed the construction of utility curves of negative values. Do not integrate renewable 
Table 3. Strategy $A_{3}, A_{4}$ and $A_{3}^{\prime}, A_{4}^{\prime}$

\begin{tabular}{l|c|c|c|c|}
\hline P.risk averse & & $\lambda=\mathbf{2}$ & & $=-\mathbf{2}$ \\
\hline Strategy & $A_{1}$ & $A_{2}$ & $A_{1}^{\prime}$ & $A_{2}^{\prime}$ \\
\hline $\mathbf{u}(\mathbf{E}(\mathbf{x}))$ & 0.5201 & 0.5619 & 0.8890 & 0.9045 \\
$\mathbf{E ( u ( x ) )}$ & 0.8558 & 0.8572 & 0.5860 & 0.5753 \\
\hline
\end{tabular}

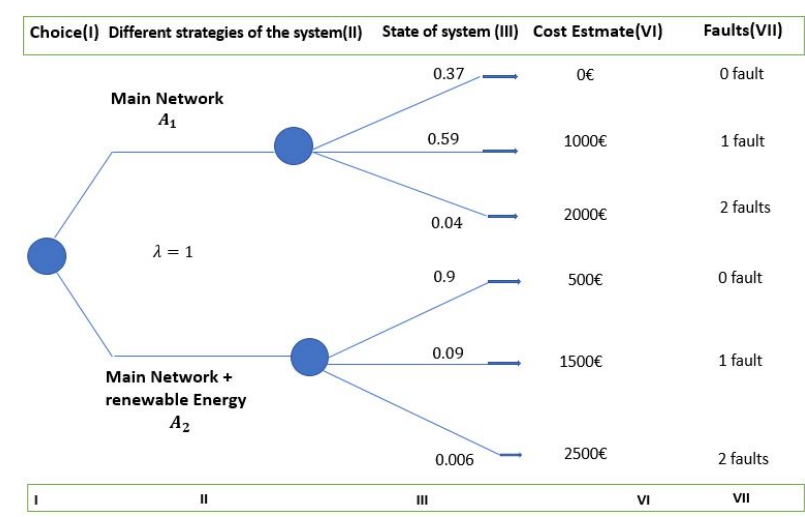

Figure 5. Sansibility lottery

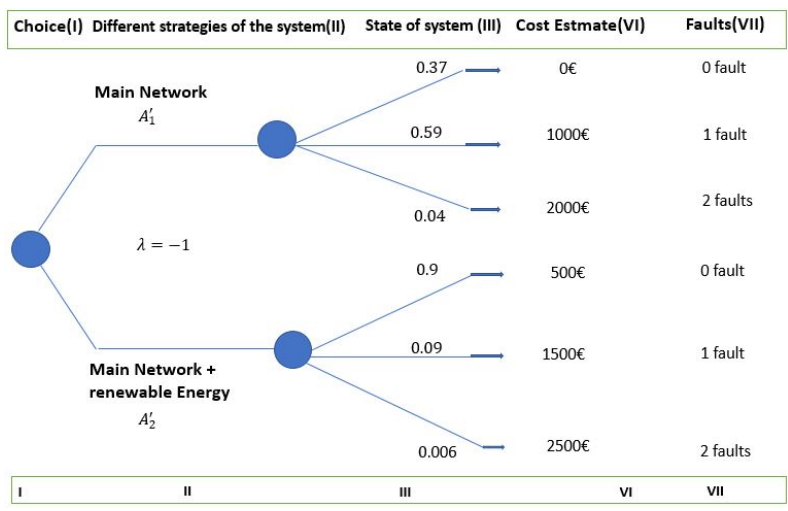

Figure 6. Sansibility lottery

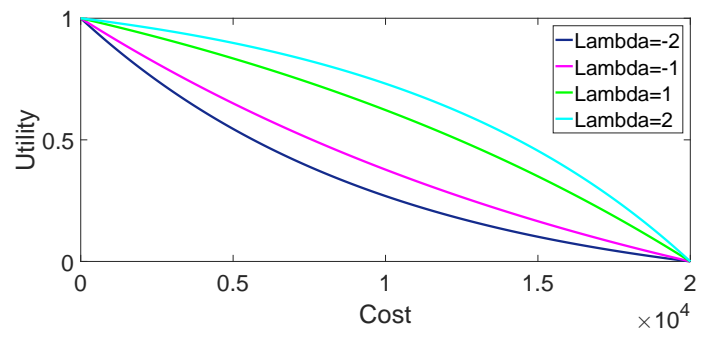

Figure 7. Attitudes towards risk(utility curve): The concave utility curve has diminishing marginal utility, is associated with risk-averse behavior, while the convex curve exhibits an increasing marginal utility reflecting a risky (risky) risk behavior.For $\lambda=2$, resp. $\lambda=1$, the concave curves attests a decision maker with an aversion to risk. For $\lambda=-2$, resp. $\lambda=-1$, the convex curves attest to a decision-maker with a predilection for risk. 
production exposes to risk which is reduced by a preventive approach but which entails an investment cost.

The decision maker finally chooses and above all the hope that maximizes its utility. In the case of this work the decision maker chooses the strategy by setting the $\lambda$ dislike parameter to 3 and -3 . This is to say that the theory of choice is based on hope. This expectation is calculated for all the alternatives, i.e the different lotteries, and then we choose the one that maximizes the expected utility of the final wealth. The results suggest a differentiation of attitudes towards risk which, in turn, influences a choice of decision options.

\section{References}

277

1. Grechuk, B.; Zabarankin, M. Risk averse decision making under catastrophic risk. European Journal of Operational Research 2014, 239, 166-176.

2. Tyrrell Rockafellar, R.; Royset, J.O. Engineering decisions under risk averseness. ASCE-ASME Journal of Risk and Uncertainty in Engineering Systems, Part A: Civil Engineering 2015, 1, 04015003.

3. Bogdan, G.; Michael, Z. Risk averse decision making under catastrophic risk. ScienceDirect 2014.

4. Alvehag, K. Risk-based methods for reliability investments in electric power distribution systems. PhD thesis, KTH Royal Institute of Technology, 2011.

5. Öztürké, M.; Tsoukiàs, A.; Vincke, P. Preference modelling. In Multiple criteria decision analysis: State of the art surveys; Springer, 2005; pp. 27-59.

6. $\quad$ Alain, S. Décider sur plusieurs critères. Presses Polytechniques romandes 1985.

7. Greco, S.; Figueira, J.; Ehrgott, M. Multiple criteria decision analysis. Springer's International series 2005.

8. John, v.N.; Oskar, M. Theory of Games and Economic Behavior; Princeton University Press, 1944.

9. Von Neumann, J.; Morgenstern, O. Theory of games and economic behavior; Princeton university press, 2007.

10. Gayant, J.P. L'apport des modèles non-additifs en théorie de la décision dans le risque et l'incertain. Revue française d'économie 1998, 13, 199-227.

11. Khazaii, J. Energy-efficient HVAC Design: An Essential Guide for Sustainable Building; Springer, 2014.

12. Gritsevskyi, A.; Nakićenovi, N. Modeling uncertainty of induced technological change. Energy policy $2000,28,907-921$.

13. Doukas, H.C.; Andreas, B.M.; Psarras, J.E. Multi-criteria decision aid for the formulation of sustainable technological energy priorities using linguistic variables. European Journal of Operational Research 2007, 182, 844-855.

14. Hossain, J.; Mahmud, A. Renewable energy integration: challenges and solutions; Springer Science \& Business Media, 2014.

15. Kumar, A.; Sah, B.; Singh, A.R.; Deng, Y.; He, X.; Kumar, P.; Bansal, R. A review of multi criteria decision making (MCDM) towards sustainable renewable energy development. Renewable and Sustainable Energy Reviews 2017, 69, 596-609.

16. Henao, F.; Cherni, J.A.; Jaramillo, P.; Dyner, I. A multicriteria approach to sustainable energy supply for the rural poor. European Journal of Operational Research 2012, 218, 801-809.

17. Azzopardi, B.; Martinez-Cesena, E.A.; Mutale, J. Decision support system for ranking photovoltaic technologies. IET Renewable Power Generation 2013, 7,669-679.

18. Chedid, R.; Akiki, H.; Rahman, S. A decision support technique for the design of hybrid solar-wind power systems. IEEE transactions on Energy conversion 1998, 13, 76-83.

19. Cherni, J.A.; Dyner, I.; Henao, F.; Jaramillo, P.; Smith, R.; Font, R.O. Energy supply for sustainable rural livelihoods. A multi-criteria decision-support system. Energy Policy 2007, 35, 1493-1504.

20. Li, Y. Decision making under uncertainty in power system using Benders decomposition 2008.

21. Martinez-Cesena, E.A. Real options theory applied to renewable energy generation projects planning 2012.

22. Rose, B. Risk aversion in maintenance: overmaintenance and the principal-agent problem. IMA Journal of Management Mathematics Advance Access published July 4, 20052005.

23. Oleg, K. The Utility Theory in Maintenance and Repair Strategy. The 2nd International Conference on Rehabilitation and Maintenance in Civil Engineering, Procedia Engineering 54 ( 2013 ) 604 - 6142013.

24. Pradier, P.C.; et al. La notion de risque en économie. Technical report, 2006.

25. Cha, E.J.; Ellingwood, B.R. The role of risk aversion in nuclear plant safety decisions. Structural Safety 2013 , $44,28-36$.

26. Cha, E.J.; Ellingwood, B.R. Risk-averse decision-making for civil infrastructure exposed to low-probability, high-consequence events. Reliability Engineering E System Safety 2012, 104, 27-35.

27. Bruno, S.; Ahmed, S.; Shapiro, A.; Street, A. Risk neutral and risk averse approaches to multistage renewable investment planning under uncertainty. European Journal of Operational Research 2016, 250, 979-989.

28. IAEA. Expansion planning for electrical generating systems: A Guidebook; International Atomic Energy Agency, 1984. 